\title{
Avaliação da atividade genotóxica, citotóxica e antimicrobiana da infusão das folhas de Spondias purpurea $\mathrm{L}$.
}

\author{
Evaluation of genotoxic activity, cytotoxic and antimicrobial infusion of Spondias purpurea L. \\ leaves
}

\author{
R. S. Santos, R. X. Santos \& G. Marisco*
}

Departamento de Ciências Naturais, Universidade Estadual do Sudoeste da Bahia, Estrada do Bem-Querer, Km 4, s/n, Bairro Universitário, 45.083-900, Vitória da Conquista, Bahia, Brasil

*gabrielemarisco@uesb.edu.br

(Recebido em 20 de maio de 2016; aceito em 5 de março de 2017)

\begin{abstract}
O uso de plantas medicinais cada vez mais é mostrado como uma fonte alternativa para tratamento de doenças. Este trabalho visou determinar o nível genotóxico e citotóxico da infusão das folhas de Spondias purpurea L., investigar o potencial antimicrobiano e conhecer o perfil cromatográfico de compostos presentes na infusão. Foram realizados os testes Allium cepa e Artemia salina para avaliar a genotoxicidade e citotoxicidade, respectivamente. Os métodos de difusão em disco e concentração inibitória mínima (CIM) foram usados para determinar a atividade antibacteriana nas bactérias Staphylococcus epidermidis, $S$. aureus, Escherichia coli, Salmonella enterica sorotipo Choleraesuis, Pseudomonas aeruginosa e Klebsiella pneumoniae. No teste de A. cepa, a análise estatística não indicou diferenças significativas do índice mitótico entre os tratamentos e o controle. $\mathrm{O}$ teste de $A$. salina mostrou que a infusão apresentou toxicidade frente aos náuplios a partir da dose $10 \mu \mathrm{g} / \mathrm{mL}$. Em relação à atividade antibacteriana, houve presença de halos de inibição contra as bactérias $S$. aureus e $E$. coli, e quando submetidos ao CIM, a infusão apresentou atividade antibacteriana contra todas as bactérias testadas. Sendo assim, a partir desse estudo pode-se inferir que a infusão (chá) das folhas da planta S. purpurea se mostrou como uma fonte medicinal não genotóxica para o teste A. cepa, bem como uma possível fonte antitumoral indicada pelo teste A. salina, além de uma promissora fonte antibacteriana.

Palavras-chaves: Genotoxicidade, Antimicrobiana, Seriguela.
\end{abstract}

The use of medicinal plants is increasingly shown as an alternative source for the treatment of diseases. This study aimed to determine the level genotoxic and cytotoxic infusion of the leaves of Spondias purpurea L., study the antimicrobial potential and know the chromatographic profile of the compounds present in the infusion. Tests were performed Allium cepa and Artemia salina to assess the genotoxicity and cytotoxicity, respectively. The disk diffusion method and the minimum inhibitory concentration (MIC) they were used to determine the antibacterial activity in bacteria Staphylococcus epidermidis, S. aureus, Escherichia coli, Salmonella enterica serotype Choleraesuis, Pseudomonas aeruginosa e Klebsiella pneumoniae. On test A. сера, statistical analysis indicated no significant differences in mitotic index between treatment and control. The test of A. salina showed that infusion toxicity has opposite from the nauplii dose $10 \mathrm{mg} / \mathrm{ml}$. With regard to antibacterial activity, there was the presence of halos of inhibition against the bacteria S. aureus e E. coli, and when subjected to MIC infusion showed antibacterial activity against all tested bacteria. Thus, from this study can be inferred that the infusions (tea) from the plant leaves $S$. purpurea showed as a non-genotoxic medicinal source for testing A. cepa, as well as a possible antitumor source indicated by the test $A$. salina, as well as a promising antibacterial source.

Keywords: Genotoxicity, Antimicrobial, Seriguela.

\section{INTRODUÇÃO}

A utilização de plantas medicinais pela população é uma cultura antiga, a partir das primeiras civilizações perceberam o poder curativo das plantas, a qual foi por muitos anos o único recurso terapêutico utilizado para tratar doenças [1]. De acordo com a Agência Nacional de Vigilância Sanitária - ANVISA (2012) [2], as plantas medicinais atuam como remédios capazes de aliviar 
ou ainda curar enfermidades, sendo utilizadas e documentadas há séculos pela população, inclusive fornecida pelo Sistema Único de Saúde (SUS), desde a década de 80. As plantas medicinais têm sido utilizadas para diversos tipos de tratamento, como diabetes [3], hipertensão [4], obesidade [5], doenças causadas por fungos e bactérias [6], entre outras. Diante o crescente número de usuários da medicina popular, torna-se importante aumentar as informações sobre plantas medicinais, principalmente no que diz respeito à suas atividades curativas e ação toxicológica.

A forma de uso mais utilizada de plantas medicinais é em chá, através da infusão ou decocção, a depender da característica da planta e da parte que está sendo utilizada [7]. Albertasse et al. (2010) [8] alerta quanto ao uso do método de decocção, pois a depender da planta pode haver degradação ou eliminação dos princípios ativos da mesma, inativando o efeito terapêutico do chá ou tornando-o perigoso à saúde. A toxidade presente em algumas plantas medicinais vem sendo retratada em diversos trabalhos, e pode ser acentuada com a forma de uso, bem como a dosagem utilizada pela população [9], podendo ser evitada desde que as plantas sejam consumidas da maneira e na dosagem correta [10].

As espécies vegetais são capazes de sintetizar substâncias com alguma atividade biológica, os metabólitos secundários. Esses compostos são muito utilizados de forma direta ou indireta por indústrias farmacêuticas, alimentícias, cosméticas e agroquímicas. Os metabólitos são classificados a partir de sua composição química e suas funções, como por exemplo, para os tratamentos antioxidante, antibacteriano, anti-inflamatório, antitumoral, entre outros [11, 12].

A família Anacardiaceae abrange cerca de setenta gêneros, distribuídas em diversas regiões do mundo. Esta família é bem conhecida pela presença de fenóis e ácidos fenólicos [13]. Nos países de clima temperado o gênero Spondias é o segundo gênero mais importante, possuindo 18 espécies descritas, seis dessas ocorrem no Brasil, são árvores frutíferas tropicais em domesticação e exploradas pelo seu valor comercial [14]. A espécie Spondias purpurea L. (seriguela) é distribuída principalmente no nordeste brasileiro e tem sido utilizada contra diversas doenças e sintomas como diabetes, hipertensão, colesterol, diarreia e dores estomacais [15, 16, 17], entretanto, poucos estudos de toxicidade e mutagenicidade dessa planta foram encontrados na literatura [13].

Para avaliar a genotoxicidade das plantas, o teste com raízes de Allium cepa é uma alternativa barata e apresenta bons resultados para análises de alterações cromossômicas e mitóticas [10]. Além disso, têm sido utilizados em estudos com agentes químicos [18], ambientais [19] e com extratos de plantas [20] para avaliação genotóxica. O ensaio de letalidade com Artemia salina abrange desde potencial tóxico de plantas, possível bioatividade, screening biológico até etapas precursoras de pesquisas in vivo [21]. Neste contexto, esse trabalho visou verificar a genotoxicidade e citotoxicidade da infusão de folhas de $S$. purpurea através dos bioensaios $A$. cepa e A. salina, bem como investigar o seu potencial antibacteriano.

\section{MATERIAL E MÉTODOS}

As folhas de $S$. purpurea foram coletadas na cidade de Brumado, Bahia, Brasil com localização geográfica definida com Latitude $=14^{\circ} 12^{\prime} 13^{\prime}$ 'S e Longitude $=41^{\circ} 39^{\prime} 55^{\prime}$ ' W. A coleta foi realizada no mês de Setembro de 2013 e uma amostra testemunha foi identificada e incorporada ao herbário HUESBVC da UESB.

As folhas foram desidratadas em estufa de circulador de ar e os chás das folhas foram preparados pelo método de infusão, ficando cobertas por $15 \mathrm{~min}$ em água destilada fervente. A infusão foi resfriada a temperatura ambiente.

No bioensaio de genotoxicidade bulbos de $A$. серa enraizados foram transferidos para as concentrações de 25,50 e $100 \mathrm{mg} / \mathrm{mL}$ da infusão de $S$. purpurea, onde as raízes ficaram submersas por $24 \mathrm{~h}$. Como controle negativo foi utilizado a água destilada. Para cada concentração da infusão foram dispostos quatro bulbos de A. cepa seguindo a metodologia proposta por Sturbelle et al. (2010) [22]. Foram analisadas 3500 células de cada bulbo, totalizando 14000 células por tratamento. As lâminas foram avaliadas observando-se as células em intérfase, prófase, metáfase, anáfase e telófase, com auxílio de microscópio óptico com a objetiva de 100X. Foram calculados os valores médios do número de células de cada uma das fases do ciclo celular de A. cepa, e 
determinado o índice mitótico (IM) em porcentagem pelo número de células em divisão dividido pela soma do número das células em interfase e das células em divisão [23]. Para o índice mitótico a análise estatística dos dados foi realizada pelo teste $\mathrm{F}$ com nível de probabilidade $<0.05$ considerado significativo. Para o número de células em cada fase mitótica os dados foram analisados pelo teste estatístico ANOVA seguido pelo teste Tukey, onde o valor $p<0.05$ foi considerado significativo.

O bioensaio de A. salina para citotoxicidade foi realizado de acordo com a metodologia proposta por Meyer et al. (1982) [24]. Um total de 0,02 g de ovos de A. salina foram colocados em $300 \mathrm{~mL}$ de solução salina a 3,5\% e pH 8-8,5 por 25 horas até a eclosão. Foram coletados 10 náuplios, individualmente, com o auxílio de uma pipeta e transferido para cada poço da placa de 24 poços. Foram feitas diluições da infusão de $S$. purpurea em água salina para obtenção das concentrações de $0,001,0,01,0,1 \mathrm{e} 1 \mathrm{mg} / \mathrm{mL}$. Como controles foram usados água salina $(3,5 \%)$ e dicromato de potássio $(0,104 \mathrm{~g} / \mathrm{L})$. Todas as concentrações foram testadas em triplicata. O número de óbitos foi contado após 24 horas de exposição e a $\mathrm{CL}_{50}$ (concentração letal) determinada. Para determinar a toxicidade, foi utilizada a escala proposta por Meyer et al. (1982) [24], que descreveram uma escala onde os valores de $\mathrm{CL}_{50}<500 \mu \mathrm{g} / \mathrm{mL}^{-1}$ indicam toxicidade, $\mathrm{CL}_{50}$ entre 500 e $1000 \mu \mathrm{g} / \mathrm{mL}^{-1}$ denotam toxicidade moderada, enquanto $\mathrm{CL}_{50}>1000 \mu \mathrm{g} / \mathrm{mL}^{-1}$ sugerem ausência de toxicidade.

Para a realização da atividade antibacteriana foram utilizados os métodos de difusão em Ágar e Concentração inibitória mínima (CIM) em triplicata. Foram utilizadas as bactérias Grampositivas: S. epidermidis (ATCC35984) e S. aureus (ATCC25921) e Gram-negativas: E. coli, $S$. enterica sorotipo Choleraesuis, P. aeruginosa (ATCC27853) e K. pneumoniae. As bactérias foram inoculadas em meio de cultura Ágar Müeller-Hinton, incubadas a $37^{\circ} \mathrm{C}$ por 24 horas e ajustadas para concentração $1-2 \times 10^{8}$ células $\mathrm{mL}^{-1}$.

Para o teste de difusão em Ágar foi inoculado na placa contendo meio de cultura Ágar MüellerHinton $100 \mu \mathrm{L}$ da suspensão bacteriana, em seguida foram produzidos pequenos poços no meio de cultura com capacidade para $50 \mu \mathrm{L}$ adaptado de Porfírio et al. (2009) [25]. E em cada poço foi adicionado $40 \mu \mathrm{L}$ da infusão filtrada $(0,22 \mu \mathrm{m})$ nas concentrações de $1,50,100$ e $200 \mathrm{mg} / \mathrm{mL}$. Como controle positivo foi utilizado cloridrato de tetraciclina $(0,02 \mathrm{mg} / \mathrm{mL})$. As placas foram mantidas a $37{ }^{\circ} \mathrm{C}$ durante $24 \mathrm{~h}$ e a determinação da atividade antimicrobiana foi medida em milímetros de acordo com o halo de inibição do crescimento bacteriano.

As CIMs foram determinadas por ensaios de microdiluições em placas de 96 poços, como sugerido pelas normas CLSI (2011) [26]. Colônias individuais de culturas bacterianas crescidas em meio ágar Mueller-Hinton foram diluídas em solução salina e ajustadas para concentração 1.5 $\times 10^{8} \mathrm{UFC} / \mathrm{mL}$. Em seguida, as suspensões bacterianas foram diluídas em caldo Mueller-Hinton e plaqueadas nas placas de 96 poços com densidade igual a $5.0 \times 10^{5} \mathrm{UFC} /$ cels. Diferentes concentrações do chá de $S$. purpurea $(200,100,50,25,12,5,6,25$ e $1 \mathrm{mg} / \mathrm{mL})$ foram adicionados a cada poço, em seguida as placas foram incubadas a $37^{\circ} \mathrm{C}$ por $24 \mathrm{~h}$, e determinada a CIM. Foram utilizados água destilada e cloridrato de tetraciclina $(0.02 \mathrm{mg} / \mathrm{mL})$ como controles. Controles de viabilidade dos microrganismos testados e da esterilidade do meio de cultura também foram realizados.

As análises fitoquímicas foram realizadas por Cromatografia em Camada Delgada (CCD) em placas cromatográficas de alumínio TLC de sílica gel 60 F 254 (Merck, Darmstadt, Alemanha). Foram aplicados nas placas alíquotas de aproximadamente $10 \mu \mathrm{L}$ da infusão de $S$. purpurea utilizando sistemas eluentes. O sistema A, constituído de ácido acético e clorofórmio (1:9), foi utilizado para a separação de fenóis. O sistema B, hexano e clorofórmio (3:2), para separar fenil propenos. O sistema C, BAW - butanol, ácido acético e água (4:1:5), para flavanoides. O sistema D, BAW (14:1:5), para separar taninos. O sistema E, clorofórmio, metanol e água (13:7:2), para separar sapogeninas. O sistema F, metanol e hidróxido de amônio (200:3), para alcaloides. Por último, o sistema G, BAW (4:1:5), para separar açúcares. Os cromatogramas foram desenvolvidos em cubas saturadas e revelados com auxílio da em luz ultravioleta de 254 e $356 \mathrm{~nm}$. 


\section{RESULTADOS E DISCUSSÃO}

Neste estudo, foi possível observar que a exposição das células de A. cepa quando submetidas à infusão de $S$. purpurea não indicaram diferenças significativas no índice mitótico (Tabela 1), mostrando que $S$. purpurea nestas condições não apresenta efeitos genotóxicos. Estudos realizados por Atoyebi et al. (2015) [27] observaram que extratos aquosos de uma outra espécie do gênero Spondias, a planta Spondias mombin também não apresentou efeito genotóxico frente as células de A. серa. É importante destacar que com o uso cada vez mais frequente de plantas medicinais, são necessários estudos de toxicidade, e o teste $A$. cepa é umas das primeiras análises para o monitoramento in situ da genotoxicidade de substâncias ambientais [28, 9].

Tabela 1: Número de células da raiz de A. cepa contadas em diferentes concentrações da infusão de $S$.

purpurea.

\begin{tabular}{cccccc}
\hline Tratamentos & Total de células & $\begin{array}{c}\text { Células em } \\
\text { interfase }\end{array}$ & $\begin{array}{c}\text { Células em } \\
\text { divisão }\end{array}$ & IM (\%) & $\mathrm{F}<0,05$ \\
\hline Controle & 14000 & 12643 & 1357 & $9,70^{\text {a }}$ & - \\
$25 \mathrm{mg} / \mathrm{ml}$ & 14000 & 12965 & 1035 & $7,39^{\text {a }}$ & 0,95 \\
$50 \mathrm{mg} / \mathrm{ml}$ & 14000 & 12862 & 1138 & $8,13^{\mathrm{a}}$ & 0,96 \\
$100 \mathrm{mg} / \mathrm{ml}$ & 14000 & 12782 & 1218 & $8,70^{\text {a }}$ & 0,97
\end{tabular}

Controle = água destilada; $\mathrm{IM}=$ índice mitótico; Os valores não diferem estatisticamente pelo teste $\mathrm{F}$.

No entanto, a exposição à infusão de $S$. purpurea causou alterações nas frequências da fase celular anáfase nas concentrações de 25 e $50 \mathrm{mg}$ (Tabela 2) quando comparadas com o controle. De acordo com Parsons, Williams (2000) [29] os alcaloides, têm propriedades biológicas, incluindo a inibição da síntese de proteínas e DNA em certas células. Colocando-os assim como possíveis promotores da condensação dos cromossomos em fases do ciclo mitótico [30].

Tabela 2: Número de células em cada fase mitótica da raiz de Allium cepa presentes nas diferentes concentrações da infusão de Spondias purpurea.

\begin{tabular}{cccccc}
\hline \multirow{2}{*}{ Tratamentos } & Total de Células & \multicolumn{3}{c}{ Número de células em divisão celular } \\
\cline { 3 - 6 } & & 871 & 192 & 130 & 164 \\
& 14000 & 584 & 340 & $25^{*}$ & 86 \\
Controle & 14000 & 611 & 414 & $31^{*}$ & 82 \\
$25 \mathrm{mg} / \mathrm{ml}$ & 14000 & 598 & 472 & 54 & 92 \\
$50 \mathrm{mg} / \mathrm{ml}$ & 14000 & & Metáfase & Anáfase & Telófase \\
$100 \mathrm{mg} / \mathrm{ml}$ &
\end{tabular}

* Significativamente diferente do tratamento controle pelo teste de Tukey $(\mathrm{p}<0,05 \%)$.

Controle $=$ água destilada

Em relação aos resultados do bioensaio com A. salina, foi possível mostrar que a infusão das folhas de $S$. purpurea apresentou toxicidade frente aos náuplios. O $\mathrm{CL}_{50}$ foi de $10 \mu \mathrm{g} / \mathrm{mL}-1 \mathrm{em}$ $24 \mathrm{~h}$ de exposição, valor altamente tóxico quando comparado a escala proposta por Meyer et al. (1982) [24]. Este bioensaio de citotoxicidade tem sido utilizado para seleção de extratos de plantas com substâncias de potencial farmacológico. Além disso, este teste possui correlação significativa com a inibição de crescimento de tumor sólido em linhagens de células humanas, que tem sido demonstrado pelo Instituto Nacional do Câncer no EUA, sugerindo o valor deste bioensaio como uma ferramenta de pré-triagem para pesquisa de drogas antitumorais [31].

Estudos de toxicidade com as folhas da planta S. purpurea foram relatadas por Dantas (2012) [32], que realizou o bioensaio com extrato etanólico das folhas e observou presença de toxicidade moderada após $48 \mathrm{~h}$ de exposição com valores de $\mathrm{CL}_{50}$ igual a $823 \mu \mathrm{g} / \mathrm{mL}^{-1}$. E estudos realizados por Fonseca et al. (2013) [33] mostraram que a toxicidade pode variar a depender da parte da 
planta que está sendo analisada, pois não foi observado toxicidade no extrato aquoso da semente de S. purpurea, apresentando $100 \%$ de sobrevivência na maior concentração testada que foi na de dose de $500 \mu \mathrm{g} / \mathrm{mL}^{-1}$. Dessa forma, os metabólitos secundários podem ou não serem tóxicos, fazendo-se necessário a realização de estudos de toxicidade em plantas utilizadas medicinalmente.

Quanto ao bioensaio antibacteriano da infusão das folhas de S. purpurea, o método de difusão em disco forneceu dados qualitativos, com a presença de halos de inibição contra as bactérias $S$. aureus e E. coli, nas concentrações de 100 e $200 \mathrm{mg} / \mathrm{ml}$ (Tabela 3). As doses testadas que apresentaram inibição antibacteriana são semelhantes às doses utilizadas na forma de infusão pelos consumidores caseiros desta planta, isto é relevante e se destaca, mostrando que de fato o uso através de chás pode ser eficaz para problemas gastrointestinais como descrito na literatura $[15,17]$.

Tabela 3: Diâmetro dos halos de inibição ( $\mathrm{mm})$ da infusão das folhas de Spondias purpurea.

\begin{tabular}{lccccc}
\hline & \multicolumn{5}{c}{ Tratamentos em mg/mL } \\
\cline { 2 - 6 } \multicolumn{1}{c}{ Bactérias } & 1 & 50 & 100 & 200 & Controle Positivo \\
\hline Staphylococcus. epidermidis & 0 & 0 & 0 & 0 & 31 \\
Staphylococcus aureus & 0 & 0 & 4 & 6 & 31 \\
Escherichia coli & 0 & 0 & 0 & 4 & 20 \\
Salmonella enterica sorotipo & 0 & 0 & 0 & 0 & 22 \\
Choleraesuis & & & & & 9 \\
Pseudomonas aeruginosa & 0 & 0 & 0 & 0 & 7 \\
Klebsiella pneumoniae & 0 & 0 & 0 & 0 & \\
\hline Conyyyyy
\end{tabular}

Controle positivo: Cloridrato de tetraciclina $(0,02 \mathrm{mg} / \mathrm{mL})$.

No teste CIM não foi observado inibição total da infusão das folhas de $S$. purpurea sobre as bactérias testadas, no entanto, nas concentrações de 25 e $12,5 \mathrm{mg} / \mathrm{ml}$ foi notado a inibição parcial $\left(\mathrm{CL}_{50}\right)$ sobre as bactérias Gram-positivas: S. epidermidis e S. aureus e Gram-negativas: E. coli, $S$. enterica sorotipo Choleraesuis e K. pneumoniae (Tabela 4). O fato do CL ter sido 50 pode estar relacionado ao fato do teste ter sido realizado com a infusão das folhas de $S$. purpurea e não com extratos mais isolados.

Tabela 4: Valores de CIM e CL ${ }_{50}$ do CIM da infusão de Spondias purpurea.

\begin{tabular}{lcc}
\hline \multicolumn{1}{c}{ Bactérias } & \multicolumn{2}{c}{ Concentrações em mg/mL } \\
\cline { 2 - 3 } & CIM & CL 50 \\
\hline Staphylococcus. epidermidis & - & 25 \\
Staphylococcus aureus & - & 25 \\
Escherichia coli & - & 25 \\
Salmonella enterica sorotipo Choleraesuis & - & 25 \\
Klebsiella pneumoniae & - & 12,5 \\
\hline
\end{tabular}

- não apresentou CIM

Extratos das folhas de $S$. purpurea já foram relatados na literatura com atividade inibitória contra as bactérias E. coli, S. enterica sorotipo Typhi e Shigella flexneri [34, 35]. Miranda-Cruz et al. (2012) [36] consideraram a $S$. purpurea como uma fonte antimicrobiana promissora, já que os extratos etanólicos da folha desta planta apresentaram uma zona de atividade antimicrobiana elevada contra Bacillus cereus, bactéria causadora de intoxicação alimentar. Alencar et al. (2015) [37] constatou em seus estudos que o extrato bruto das folhas de $S$. purpurea combinado com o 
antibiótico eritromicina apresentou efeito inibitório significativamente maior com doses menores contra quatro cepas distintas de $S$. aureus, quando comparados ao antibiótico ou extrato vegetal testados isoladamente.

Outras plantas da família Anacardiaceae têm sido avaliadas sobre sua ação antimicrobiana, Spondias dulcis e Schinopsis brasiliensis foram avaliadas com relação a essa atividade seguindo a mesma metodologia deste estudo, e mostraram-se ativas contra as bactérias $S$. aureus e E. coli [38].

Há uma disposição mundial quanto ao uso de antimicrobianos naturais no combate a microrganismos causadores de doenças $[39,40]$. Neste sentindo, tanto $S$. aureus que pode provocar doenças, que vão desde uma simples infecção (espinhas, furúnculos e celulites) até infecções graves (pneumonia, meningite, endocardite, síndrome do choque tóxico, septicemia e outras) [41], E.coli que pode ocasionar doenças em superfícies mucosas ou ainda se disseminar através do organismo, implicando em processos de infecções gastrointestinais, urinárias e meningite [42, 43], quanto outras bactérias patológicas avaliadas neste estudo, podem ser tratadas utilizando chás medicinais de $S$. purpurea ou associadas com antibióticos durante o tratamento, visto ser um hábito comum de muitas pessoas atualmente. Além disso, é importante destacar que as substâncias da infusão ainda não foram testadas isoladamente, o que pode aumentar ainda mais essas atividades biológicas descritas.

Brito (2010) [44] relaciona a presença de metabólitos secundários, como terpenos encontrados em S. purpurea, podendo estar relacionados com essa ação antimicrobiana, principalmente contra S. aureus [45]. Ravishankar et al. (1994) [46], e Momesso et al. (2009) [47], atribuíram o efeito antitumoral aos flavanóides, metabólito identificado em estudos com S. purpurea. A partir das CCDs realizadas neste estudo, a infusão das folhas de $S$. purpurea indicaram a presença de fenóis, flavonoides, taninos, sapogeninas e alcaloides. Estudo das cascas de $S$. purpurea realizado por Engels et al. (2012) [14], demonstram a presença dos ácidos fenólicos e flavanoides como quercetina, canferol, canferídeo e raminetina. Os óleos essenciais das folhas de S. purpurea foram descritos por Brito (2010) [44] com a presença de terpenos importantes como $\beta$-cariofileno e $\alpha$ humuleno, os quais têm sido relatados como uma fonte antitumoral promissora [48].

Além disso, conforme Silva et al. (2014) [49], em diversas regiões do mundo, espécies do gênero Spondias têm sido utilizados para diversos tratamentos, e muitas dessas ações foram atribuídas aos compostos fenólicos (taninos e flavonoides), na maioria, presentes nas folhas.

\section{CONCLUSÃO}

A presente investigação mostrou que a infusão das folhas de $S$. purpurea não apresentou genotoxicidade frente às células de $A$. cepa, no que se refere ao aumento ou diminuição do índice mitótico. Entretanto, pode-se inferir que a planta em estudo exibe promissor potencial de atividade antitumoral, visto que no teste de A. salina apresentou-se altamente ativa em concentrações baixas e a análise fitoquímica da infusão foi positiva para a presença de terpenos e flavanoides, metabólitos associados à ação antitumoral.

A infusão de $S$. purpurea apresentou-se também como uma fonte antimicrobiana no teste de halo de inibição contra duas bactérias patogênicas, $S$. aureus e E. coli, e no teste CIM demostrou atividade antibacteriana para as bactérias S. epidermidis, S. aureus, E. coli, S. enterica sorotipo Choleraesuis e $K$. pneumoniae. Essas atividades podem estar relacionadas com os metabólitos secundários (fenóis, flavonoides, taninos, sapogeninas e alcaloides) encontrados no chá na forma de infusão, método mais habitualmente usado.

Neste contexto, a espécie $S$. purpurea mostrou-se como uma fonte medicinal promissora, contudo, são necessários isolamento de metabólitos, estudos fitoquímicos e biológicos para determinação do potencial antimicrobiano e antitumoral.

\section{AGRADECIMENTOS}

À Universidade Estadual do Sudoeste da Bahia; à estagiária do laboratório de Biologia Geral Marina Amorim e ao Prof. Dr. Avaldo Soares Filho que realizou a identificação da espécie, responsável pelo Herbário. 


\section{REFERÊNCIAS BIBLIOGRÁFICAS}

1. Badke MR, Budó MLD, Silva FM, Ressel LB. Plantas medicinais: O saber sustentando na prática do cotidiano popular. Esc Enfer Anna Nery. 2011 Mar;15(1):132-139.

2. Anvisa. Agência Nacional de Vigilância Sanitária. Nota técnica do comitê nacional de plantas medicinais e fitoterápicos a respeito do que foi veiculado sobre plantas medicinais e fitoterápicos na imprensa televisiva e escrita no último mês. Medicamentos Fitoterápicos. 2012; Disponível em: http://www.anvisa.gov.br. Acesso em fev.

3. Dallaqua B, Damasceno DC. Comprovação do efeito antioxidante de plantas medicinais utilizadas no tratamento do Diabetes mellitus em animais: artigo de atualização. Rev Bras Pl Med. 2011 Jan;13(3):367373.

4. Lopes GAD, Feliciano LM, Diniz RES, Alves MQF. Plantas medicinais: indicação popular de uso no tratamento de hipertensão arterial sistêmica (has). Rev Ciênc Ext. 2010;6(2):143-155.

5. Souza SP, Pereira LLS, Souza AA, Santos CD. Seleção de extratos brutos de plantas com atividade antiobesidade. Rev Bras Pl Med. 2012;14(4):643-648.

6. Aneja KR, Sharma C, Joshi R. Antimicrobial activity of Terminalia arjuna Wight \& Arn.: Anethnomedicinal plant against pathogens causing ear infection. Braz J Otorhinolaryngol. 2012 Fev;78(1):68-74.

7. Vendruscolo GS, Mentz LA. Levantamento etnobotânico das plantas utilizadas como medicinais por moradores do bairro Ponta Grossa, Porto Alegre, Rio Grande do Sul, Brasil. Iher Sér Bot. 2006 Dez;61(1/2):83-103.

8. Albertasse PD, Thomaz LD, Andrade MA. Plantas medicinais e seus usos na comunidade da Barra do Jucu, Vila Velha, ES. Rev Bras Pl Med. 2010 Set;12(3):250-260.

9. Fachinetto JM, Bagantini MD, Durigon J, Silva ACF, Tedesco SB. Efeito anti-proliferativo das infusões de Achyrocline satureioides DC (Asteraceae) sobre o ciclo celular de Allium cepa. Rev Bras Farmacogn. 2007 Jan/Mar; 17(1):49-54.

10. Pinho DS, Sturbelle RT, Martino-Roth MG, Garcias GL. Avaliação da atividade mutagênica da infusão de Baccharis trimera (Less.) DC. em teste de Allium cepa e teste de aberrações cromossômicas em linfócitos humanos. Rev. bras. farmacogn. 2010 Mai;20(2):165-170.

11. Pereira RJ, Cardoso MG. Metabólitos secundários vegetais e benefícios antioxidantes. J. Biotec. Biodivers. 2012 Nov;3(4):146-152.

12. Lima Neto GA, Kaffashi S, Luiz WT, Ferreira WR, Silva YSA, Pazin GV, Violante IMP. Quantificação de metabólitos secundários e avaliação da atividade antimicrobiana e antioxidante de algumas plantas selecionadas do Cerrado de Mato Grosso. Rev bras plantas med. 2015 Jun;17(4):1069-1077.

13. Marisco G, Pungartnik, C. Spondias purpurea L. (Anacardiaceae): traditional uses, chemical composition and biological activities. Scientia Amazonia. 2015 Mai-Ago, 4 (2): 10-18.

14. Engels C, Grater D, Esquivel P, Jimenez VM, Ganzle MG, Schieber A. Characterization of phenolic compounds in jocote (Spondias purpurea L.) peels by ultra-high-performance liquid chromatography/electrospray ionization mass spectrometry. Food Res Int. 2012 Mai;46(2):557-562, doi: 10.1016/j.foodres.2011.04.003.

15. Freitas AVL, Coelho MFB, Maia SSS, Azevedo RAB. Plantas medicinais: um estudo etnobotânico nos quintais do Sítio Cruz, São Miguel, Rio Grande do Norte, Brasil. Rev Bras Bioci. 2012;10(1):48-59.

16. Nascimento JM, Conceição GM. Plantas medicinais e indicações terapêuticas da comunidade quilombola Olho D'água do Raposo, Caxias, Maranhão, Brasil. BioFar. 2011;06(2): 138-151.

17. Silva NCB, Regis ACD, EsquibeL MA, Santos JES, Almeida MZ. Uso de plantas medicinais na comunidade quilombola da Barra II - Bahia, Brasil. Bol Latinoam Caribe Plant Med Aromat. 2012 Set;11(5):435 - 453.

18. Feretti D, Zerbini I, Zani C, Ceretti E, Moretti M, Monarca S. Allium cepa chromosome aberration and micronucleus tests applied to study genotoxicity of extracts from pesticide-treated vegetables and grapes. Food Addit Contam. 2007 Jun;24(6):561-572, doi: 10.1080/02652030601113602.

19. Amaral AM, Barbério A, Voltolini JC, Barros L. Avaliação preliminar da citotoxicidade e genotoxicidade, da água da bacia do Rio Tapanhon (SP- Brasil) através do teste Allium (Allium cepa). Rev Bras Toxic. 2007;20(1/2):65-72.

20. Silva DSBS, Barboza B, Garcia ACSF, Oliveira B, Estevam CS, Neto VA, Santos ALLM, Dias AS, Scher R, Pantaleao SM. Investigation of protective effects of Erythrina velutina extract against MMS induced damages in the root meristem cells of Allium cepa. Rev Bras Farmacogn. 2013 Abr;23(2):273-278. 21. Hirota BCK, Paula CS, Miguel OG, Miguel MD. Avaliação de toxicidade in vitro: aplicabilidade do ensaio de letalidade frente á Artemia salina. Visão Acadêmica, Curitiba. 2012 Abr/Jun;13(2): 42-48, doi: 10.5380/acd.v13i2.27834. 
22. Sturbelle RT, Pinho DS, Restani RG, Oliveira GR, Garcias GL, Martino-Roth MG. Avaliação da atividade mutagênica e antimutagênica da Aloe vera em teste de Allium cepa e teste de micronúcleo em linfócitos humanos binucleados. Rev Bras Farmacogn. 2010 Jul; 20(3):409-415.

23. Pires NM, Souza IRP, Prates HT, Faria TCL, Pereira Filho IA, Magalhães PC. Efeito do extrato aquoso de leucena sobre o desenvolvimento, índice mitótico e atividade da peroxidase em plântulas de milho. Rev. Bras. Fisiol. Veg. 2001 Abr;13(1):55-65.

24. Meyer BN, Ferregni NR, Putnam JE, Jacobsen LB, Nichols DE, McLaughlin JL. Brine shrimp: a convenient general bioassay for active plant constituents. Planta Med. 1982 Mai;45(5):31-4.

25. Porfírio Z, Melo-Filho, GC, Alvino V, Lima MRF, Sant'ana AEG. Atividade antimicrobiana de extratos hidroalcoólicos de Lafoensia pacari A. St.-Hil., Lythraceae, frente a bactérias multirresistentes de origem hospitalar. Rev bras farmacogn. 2009 Set;19(3):785-789.

26. CLSI - Clinical and Laboratory Standards Institute. Performance Standards for Antimicrobial Susceptibility Testing; Twenty-First Informational Supplement. 2011 Jan;31(1): 172 p

27. Atoyebi SM, Oyeyemi IT, Dauda BA, Bakare AA. Genotoxicity and anti-genotoxicity of aqueous extracts of herbal recipes containing Luffa cylindrica (L), Nymphaea lotus (L) and Spondias mombin (L) using the Allium cepa (L) assay. Afr J Pharm Pharmacol. 2015 Abr;9(15):492-499, doi: 10.5897/AJPP2014. 4219.

28. Bagatini MD, Silva ACF, Tedesco SB. Uso do sistema teste de Allium cepa como bioindicador de genotoxicidade de infusões de plantas medicinais. Rev Bras Farmacogn. 2007 Jul/Set;17(3):444-447.

29. Parsons AF, Williams DAJ. Radical cyclisation reactions leading to polycyclics related to the Amaryllidaceae and Erythrina alkaloids. Tetrahedron 2000 Set; 56 (37): 7217-7228.

30. Silva DSBS, Garcia ACFS, Mata SS, Oliveira B, Estevam CS, Scher R, Pantaleao SM. Genotoxicity and cytotoxicity of Erythrina velutina Willd., Fabaceae, on the root meristema cells of Allium cepa. Rev Bras Farmacogn. 2011 Fev;21(1):92-97.

31. Arcanjo DDR, Albuquerque ACM, Melo-Neto Bb, Santana LCLR, Medeiros MGF, Citó AMGL. Bioactivity evaluation against Artemia salina Leach of medicinal plants used in Brazilian Northeastern folk medicine. Braz J Biol. 2012 Ago;72(3):505-509.

32. Dantas AM. Avaliação da Toxicidade e Atividade Antiulcerogênica das Folhas de Spondias purpurea L [Trabalho de conclusão de curso]. Universidade Estadual da Paraíba, Centro de Ciências Biológicas e da Saúde; 2012. 23p.

33. Fonseca RC, Souza NA, Correa TCL, Garcia LF, Reis LGV, Rodriguez AG. Assessment of toxic potential of Cerrado fruit seeds using Artemia salina bioassay. Food Sci Technol. 2013 Mai;33(2):251256.

34. Caceres A, Cano O, Samayoa B, Aquilar L. Plants used in Guatemala for the treatment of gastrointestinal disorders. 1. Screening of 84 plants against enterobacteria. J Ethnopharmacol. 1990 Ago;30(1):55-73.

35. Caceres A, Fletes L, Aguilar L, Ramirez O, Figueroa L, Taracema AM, Samayoa B. Plants used in Guatemala for the treatment of gastrointestinal disorders. 3. Confirmation of activity against enterobacteria of 16 plants. J Ethnopharmacol. 1993 Jan;38(1):31-38.

36. Miranda-Cruz E, Moreno JE, Hidalgo DC, Martinez JRV, Chavez MJA. Actividad antimicrobiana de extractos de Psidium friedrichsthalianum L., Pterocarpus hayesii L., Tynanthus guatemalensis L. y Spondias purpurea L. Bol Latinoam Caribe Plant Med Aromat. 2012 Jul;11(4):354-361.

37. Alencar LCB, Chaves TP, Santos JS, Nóbrega FP, Araújo RM, Santos VL, Felismono DC, Medeiros ACD. Efeito modulador do extrato de plantas medicinais do gênero Spondias sobre a resistência de cepas de Staphylococcus aureus à Eritromicina. Rev Ciênc Farm Básica Apl. 2015;36(1):111-116.

38. Islam SA, Ahmed KT, Manik MK, Wahid A, Kamal CSI. A comparative study of the antioxidant, antimicrobial, cytotoxic and thrombolytic potential of the fruits and leaves of Spondias dulcis. Asian Pac J Trop Biomed. 2013 Set;.3(9):682-691, doi: 10.1016/S2221-1691(13)60139-2.

39. Cruz M, Santos PO, Barbosa AMJ, Alviano DS, Trindade RC. Antifungal activity of Brazilian medicinal plants involved in popular treatment of mycoses. J Ethnopharmacol. 2007 Mai;111(2):409-12.

40. Weckesser S, Engel K, Simon-Haarhaus B, Wittmer A, Pelz K, Schempp CM. Screening of plant extracts for antimicrobial activity against bacteria and yeasts with dermatological relevance. Phytomedicine. 2007 Ago;14(7/8): 508-516.

41. Santos AL, Santos DO, Freitas CC, Ferreira BLA, Afonso IF, Rodrigues CR, Castro HC. Staphylococcus aureus: visitando uma cepa de importância hospitalar. J Bras Patol Med Lab. 2007 Dez;43(6):413-423.

42. Nataro JP, Kaper JB. Diarrheagenic Escherichia coli. Clin Microbiol Rev. 1998 Jan;11(1):142-201. 43. Sousa CP. Pathogenicity mechanisms of prokaryotic cells: An evolutionary view. Braz J Infect Dis. 2003 Fev;7(1):23-31. 
44. Brito HR. Caracterização Química de Óleos Essenciais de Spondias mombin L., Spondias purpurea L. e Spondias sp (cajarana do sertão) [Dissertação]. Universidade Federal de Campina Grande no CSTR Centro de Saúde e Tecnologia Rural; 2010.67 p.

45. Costantin MB, Sartorelli P, Limberger R, Henriques AT, Steppe M, Ferreira MJP, Ohara MT, Emerenciano VP, Kato MJ. Essential Oils from Piper cernuum and Piper regnellii: Antimicrobial Activities and Analysis by GC/MS and 13C-NMR. Planta Med. 2001 Nov;67(8):771-773.

46. Ravishankar T, Vedavalli L, Nambi AA, Selvam V, 1994. Role of tribal people in the conservation and utilizations of plant genetic resources. Madras: MSSRF.

47. Momesso LS, Moura RMX,Constantino DHJ. Atividade antitumoral do Ageratum conyzoides L. (Asteraceae). Revista Brasileira de Farmacognosia 2009; 19; (3): 660-663. http://dx.doi.org/10.1590/S0102-695X2009000500002

48. Legault J, Wivecke D, Debiton E, Pichette A, Madelmont J. Antitumor activity of balsam fir oil: Production of reactive oxygen species induced by a-humulene as possible mechanism of action. Planta Med. 2003 Mai;69(5):402-407, doi: 10.1055/s-2003-39695

49. Silva GA, Brito NJN, Santos ECG, López JA, Almeida MG. Gênero Spondias: Aspectos botânicos, composição química e potencial farmacológico. BioFar. 2014;10(1):27-41. 